PATIENT SAFETY

J.T. Wald

T.P. Maus

J.R. Geske

R.E. Carter

F.E. Diehn

T.J. Kaufmann

J.M. Morris

N.S. Murthy

K.R. Thielen

\title{
Safety and Efficacy of CT-Guided Transforaminal Cervical Epidural Steroid Injections Using a Posterior Approach
}

\begin{abstract}
BACKGROUND AND PURPOSE: Image-guided cervical transforaminal epidural injections play an important role in the management of cervical radicular pain syndromes. The safety and efficacy of these injections via an anterolateral approach has been well-studied. The goal of this retrospective review was to determine the safety and efficacy of CT-guided transforaminal epidural injections by using a posterior approach.
\end{abstract}

MATERIALS AND METHODS: Retrospective review of patient records was used to define VNPS and RMDI of patients undergoing CT-guided transforaminal cervical epidural injections between 2006 and 2010. Pain scores were recorded preprocedure, immediately postprocedure, at 2 weeks, and at 2 months. The RMDI was recorded preprocedure, at 2 weeks, and at 2 months. Data analysis of 247 patients was completed. Differences in VNPS scores and the RMDI were then compared on the basis of a CT-guided approach (anterolateral versus posterior).

RESULTS: There was no statistical difference in the degree of pain relief and improvement in the RMDI between the CT-guided transforaminal anterolateral approach and the posterior approach at 2 weeks and at 2 months. Both groups demonstrated a statistically significant improvement in pain scores and the RMDI. Approximately $35 \%$ of patients in both groups demonstrated $>50 \%$ pain relief at 2 months. There were no serious complications in either group.

CONCLUSIONS: CT-guided transforaminal cervical epidural injections by using a posterior approach are safe and effective.

ABBREVIATIONS: $\mathrm{Cl}=$ confidence interval; $\mathrm{EMG}=$ electromyography; $\mathrm{MAR}=$ missing at random; $\mathrm{RMDI}=$ Roland Morris Disability Index; VNPS = Verbal Numeric Pain Scale

$\mathbf{F}$ uoroscopic- and CT-guided transforaminal epidural steroid injections play a prominent role in the treatment of cervical radicular pain. Typically, these injections are completed via an anterolateral approach. However, numerous publications have reported rare but significant complications by using a fluoroscopic anterolateral approach. ${ }^{1-3}$ A recent study demonstrated the feasibility of a CT-guided posterior approach to cervical epidural injections. ${ }^{4}$ The aim of this retrospective study was to define the safety and efficacy of a single CT-guided cervical epidural injection by using a posterior approach.

\section{Materials and Methods}

The study was completed at a tertiary referral center in the upper Midwest after institutional review board approval. A retrospective electronic chart and radiology review was completed for patients who received cervical transforaminal epidural injections between 2006 and 2010. All patients receiving epidural injections completed a preprocedure study form, which included patient demographics, RMDI, and a VNPS: 0 , no pain; -10 , worst pain of life. Pain scores were defined immediately postprocedure in person and at 2 weeks and 2 months postprocedure by a telephone interview. RMDI was defined at 2 weeks and 2 months postprocedure by telephone interview. $\mathrm{Pa}-$ tients with unilateral radicular pain who underwent CT-guided epi-

Received May 19, 2011; accepted after revision June 29

From the Mayo Clinic, Rochester, Minnesota.

Please address correspondence to John Wald, MD, Mayo Clinic, 200 First St SW, Rochester, MN 55905; e-mail: wald.john2@mayo.edu

http://dx.doi.org/10.3174/ajnr.A2835 dural injections were included regardless of the level of cervical radiculopathy or the duration of pain. Patients who received multiple injections within a 2 -month period were excluded. Cases were defined by route of entry (anterolateral versus posterior).

\section{Procedure}

Preprocedural clinical informed consent was received from all patients. All injections were completed by experienced radiologists trained in CT-guided spine interventions. Physicians were fellowship-trained in neuroradiology or musculoskeletal radiology. Staff experience ranged from 2 years to 25 years, with an average of approximately 10 years' staff experience in CT-guided procedures. Patients were placed in a supine or prone position on the CT table at the physician's discretion. Diagnostic CT images were obtained to localize the treatment level by using a standard CT technique. Using CT guidance, a 25-ga spinal needle was placed into the lateral margin of the cervical foramen. Foraminal spread of injectate was confirmed by injection of $1-2 \mathrm{~mL}$ of diluted iodinated contrast material (diluted 1 part in 10 with preservative-free normal saline). After confirmation of periganglionic, extradural, and extravascular spread of contrast, $1 \mathrm{~mL}$ (20 mg) of lidocaine was infused. After a 2-minute observation period during which the neurologic status was assessed, $1 \mathrm{~mL}(10$ $\mathrm{mg}$ ) of dexamethasone was infused. Patients were monitored for approximately $30-45$ minutes after each procedure and then were discharged.

\section{Statistical Analysis}

The primary end point for this study was the longitudinal changes in pain and disability measured by the VNPS and RMDI. Longitudinal model-based values for both variables were obtained at each data- 


\begin{tabular}{|c|c|c|c|c|c|c|c|}
\hline \multirow[b]{2}{*}{ Approach Group } & \multicolumn{2}{|c|}{ Overall } & \multicolumn{2}{|c|}{ Anterolateral } & \multicolumn{2}{|c|}{ Posterior } & \multirow{2}{*}{$\begin{array}{c}P \\
\text { Value }\end{array}$} \\
\hline & No. & Mean & No. & Mean & No. & Mean & \\
\hline Age (yr) & 247 & $54.0 \pm 12.1$ & 77 & $53.3 \pm 11.1$ & 170 & $54.4 \pm 12.6$ & .533 \\
\hline Male (\%) & 144 & 58.3 & 96 & 62.3 & 48 & 56.5 & .386 \\
\hline Pain pre & 219 & $5.3 \pm 2.3$ & 69 & $5.4 \pm 2.3$ & 150 & $5.1 \pm 2.4$ & .373 \\
\hline Pain immediate & 246 & $1.7 \pm 1.9$ & 77 & $1.3 \pm 1.7$ & 169 & $1.8 \pm 2.0$ & .030 \\
\hline Pain 2 weeks post & 172 & $3.3 \pm 2.5$ & 50 & $3.2 \pm 2.4$ & 122 & $3.6 \pm 2.8$ & .641 \\
\hline Pain 2 months post & 120 & $2.9 \pm 2.3$ & 32 & $2.9 \pm 2.3$ & 88 & $3.0 \pm 2.5$ & .952 \\
\hline RMDI pre & 219 & $9.3 \pm 5.2$ & 69 & $9.2 \pm 5.7$ & 150 & $9.3 \pm 4.9$ & .671 \\
\hline RMDI 2 weeks post & 172 & $6.8 \pm 4.2$ & 50 & $7.0 \pm 4.2$ & 122 & $6.7 \pm 4.3$ & .613 \\
\hline RMDI 2 months post & 120 & $5.0 \pm 3.4$ & 32 & $4.9 \pm 3.4$ & 88 & $5.1 \pm 3.8$ & .969 \\
\hline
\end{tabular}

Note:- pre indicates before procedure; post, after procedure.

\begin{tabular}{|c|c|c|c|c|}
\hline & \multicolumn{2}{|l|}{ VNPS } & \multicolumn{2}{|l|}{ RMDI } \\
\hline & $\begin{array}{l}\text { Estimated Change } \\
\text { from Baseline } \\
(95 \% \mathrm{Cl})\end{array}$ & $\begin{array}{c}P \\
\text { Value }\end{array}$ & $\begin{array}{c}\text { Estimated Change } \\
\text { from Baseline } \\
(95 \% \mathrm{CI})\end{array}$ & $\begin{array}{c}P \\
\text { Value }\end{array}$ \\
\hline $\begin{array}{r}\text { Prior to } 2 \\
\text { weeks }\end{array}$ & $-2.0(-2.4$ to -1.5$)$ & $<.0001$ & $-2.5(-3.3$ to -1.7$)$ & $<.0001$ \\
\hline $\begin{array}{l}\text { Prior to } 2 \\
\text { months }\end{array}$ & $-2.1(-2.6$ to -1.6$)$ & $<.0001$ & $-3.6(-4.5$ to -2.7$)$ & $<.0001$ \\
\hline $\begin{array}{c}\text { Anterolateral } \\
\text { vs posterior }\end{array}$ & - & .832 & - & .901 \\
\hline
\end{tabular}

collection time (preprocedure, postprocedure, 2 weeks, and 2 months) by using a mixed linear model of time points and approach as fixed effects. Contrasts (statistical comparisons of model-based estimates) were configured to represent changes from baseline to 2 weeks postprocedure and baseline to 2 months postprocedure. This analysis was supplemented by using Wilcoxon rank sum scores to compare the pain scores and the RMDI value between anterolateral and posterior locations at each time point.

The percentage of change in pain scores and RMDI were calculated from preprocedure to 2 weeks and 2 months. The percentage of change in pain scores and RMDI were used to examine proportions of patients who achieved $>50 \%$ improvement, achieved complete relief (defined as a pain score of 0 ), or did not improve or worsened at each follow-up time point. $\chi^{2}$ tests were used to compare outcome proportions by procedural approach. Statistical analyses were conducted by using SAS, Version 9.2 (SAS Institute, Cary, North Carolina).

\section{Results}

A total of 285 procedures on 266 patients were reviewed, and 38 procedures (19 patients) were excluded from the dataset secondary to receiving multiple epidural steroid injections within 2 months. The final study sample included 247 patients who underwent single injections, including 77 anterolateral and 170 posterior approaches, as presented in Table 1. Preprocedural data were not available for 18 patients (11\%), and phone interview follow-up data were unavailable for 75 patients (30\%) at 2 weeks' follow-up and for 127 patients (51\%) at 2 months' follow-up.

VNPS and RMDI decreased statistically from preprocedure to both 2-week and 2-month follow-up times. Table 2 reports the estimated change from baseline in scale units. Pain scores were reduced by 2.0 (95\% CI, 1.5-2.4) and 2.1 (95\% CI,
1.6-2.6) units at 2 weeks and 2 months, respectively. Similarly for RMDI, significant reductions of 2.5 (95\% CI, 1.7-3.3) and 3.6 (95\% CI, 2.7-4.5) were identified at 2 weeks and 2 months. Estimated change with time did not depend on the procedural approach (anterolateral or posterior) for VNPS or RMDI.

Table 3 displays the response proportion based on the percentage of change from baseline in the VNPS and RMDI, along with information regarding the availability of data at followup. Overall, approximately $35 \%$ and $40 \%$ of patients who completed follow-up at 2 weeks and 2 months, respectively, achieved $>50 \%$ reduction in pain scores. Similarly approximately $23 \%$ and $35 \%$ of patients achieved $>50 \%$ improvement in the RMDI at 2 weeks and 2 months following the procedure. The VNPS was comparable between the anterolateral and posterior patient cohorts at preprocedure, 2 weeks, and 2 months. Both procedural approaches resulted in similar proportions of at least $50 \%$ improvement, complete relief, and nonimprovement or worsening for VNPS and RMDI.

The patients tolerated the procedures well. There were 4 vasovagal reactions in 247 patients. These reactions were equally divided between the anterolateral and posterior groups. None of these reactions necessitated pharmacologic intervention. No other adverse outcomes were reported.

\section{Sensitivity Analysis for Missing Data}

The impact of missing data at 2 weeks and 2 months was examined by using multiple statistical techniques. Baseline VNPS and RMDI were not significantly different between the groups of patients with 2-week data and those without 2-week data $($ VNPS, $P$ value $=.949 ;$ RMDI, $P$ value $=.975)$. However baseline VNPS and RMDI were significantly higher in the patients without 2-month data compared with patients with available 2 -month data (VNPS, $P$ value $=.002$; RMDI, $P$ value $<.001)$. Further analysis of the association of 2 -monthdata availability with the outcomes of $50 \%$ improvement, complete relief, and no improvement or worsening revealed that there was no association with outcomes at 2-week and 2-month missing-data status. This provided evidence that the 2-month missing data were MAR and that the mixed models used to estimate longitudinal changes were unbiased.

\section{Discussion}

CT-guided transforaminal cervical epidural injections by using a posterior approach are safe and effective. The efficacy of these injections is similar to that in previous reports using an anterolateral approach. 


\begin{tabular}{|c|c|c|c|c|c|}
\hline \multirow{2}{*}{$\begin{array}{l}\text { Procedure Location, } \\
\text { Response Definition }\end{array}$} & \multicolumn{3}{|c|}{ VNPS } & \multicolumn{2}{|c|}{ RMDI } \\
\hline & Immediate & 2 week & 2 month & 2 week & 2 month \\
\hline All patients & $(n=218)$ & $(n=171)$ & $(n=119)$ & $(n=171)$ & $(n=119)$ \\
\hline$>50 \%$ Improvement & $66.10 \%$ & $35.10 \%$ & $39.50 \%$ & $22.80 \%$ & $35.30 \%$ \\
\hline Complete relief & $41.06 \%$ & $16.90 \%$ & $17.50 \%$ & - & - \\
\hline No improvement/worsening & $12.84 \%$ & $30.40 \%$ & $29.40 \%$ & $36.80 \%$ & $32.80 \%$ \\
\hline Anterolateral $^{\mathrm{a}}$ & $(n=69)$ & $(n=50)$ & $(n=32)$ & $(n=50)$ & $(n=32)$ \\
\hline$>50 \%$ Improvement & $71.01 \%$ & $42.00 \%$ & $37.50 \%$ & $26.00 \%$ & $31.30 \%$ \\
\hline Complete relief & $50.65 \%^{\mathrm{b}}$ & $14.00 \%$ & $15.60 \%$ & - & - \\
\hline No improvement/worsening & $11.59 \%$ & $32.00 \%$ & $31.30 \%$ & $44.00 \%$ & $37.50 \%$ \\
\hline Posterior $^{\mathrm{a}}$ & $(n=149)$ & $(n=121)$ & $(n=87)$ & $(n=121)$ & $(n=87)$ \\
\hline$>50 \%$ Improvement & $63.76 \%$ & $32.20 \%$ & $40.20 \%$ & $21.50 \%$ & $36.80 \%$ \\
\hline Complete relief & $36.69 \%{ }^{b}$ & $18.00 \%$ & $18.20 \%$ & - & - \\
\hline No improvement/worsening & $13.42 \%$ & $29.80 \%$ & $28.70 \%$ & $33.90 \%$ & $31.00 \%$ \\
\hline
\end{tabular}

a Comparison of anterolateral vs posterior approach $P$ values $>.05$, unless indicated.

${ }^{b}$ Comparison of immediate complete relief between anterolateral and posterior approach, $P$ value $=.039$
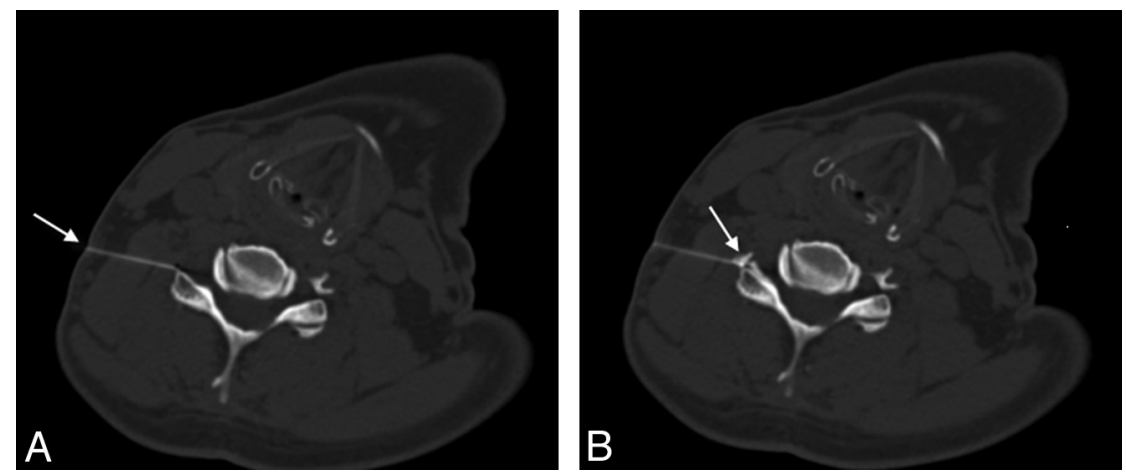

Fig 1. Anterolateral approach for CT-guided cervical epidural injection. $A$, Lateral placement of needle tip within the C6-C7 neuroforamen. $B$, Periganglionic flow and central flow of contrast into the epidural space.

Image-guided transforaminal cervical epidural steroid injections have long been a part of the therapeutic approach to cervical radiculopathy. ${ }^{5,6}$ Prospective and retrospective studies have been completed with limited patient enrollment, ${ }^{7,8}$ widely varying results, and varying time periods of followup. ${ }^{4,9-13}$ Although studies have been completed that attempted to link successful outcomes to findings on cross-sectional imaging, ${ }^{14}$ EMG findings, ${ }^{7}$ or diagnostic root blocks, ${ }^{7}$ most patients are referred for therapeutic cervical epidural injections with a variety of clinical, radiographic, and electrophysiologic data.

Although fluoroscopy has been the typical image-guided approach to transforaminal procedures, CT guidance is the preferred technique in our radiology practice and previous studies have demonstrated its safety and efficacy. ${ }^{4,10,11}$ Both an anterolateral and posterior approach have been described to access the cervical foramen (Figs 1 and 2). Given reports of complications with the fluoroscopic anterolateral approach $^{1,2,15}$ and wishing to decrease the possibility of inadvertent injection into the vertebral artery or its branches, our group has predominantly completed cervical epidural injections through a posterior approach. The assumption has been that this approach is equally as effective as the anterolateral approach completed under fluoroscopic guidance.

The results of this study support this hypothesis. The decrease in pain levels after epidural injection was statistically significant in both groups and was similar between the antero- lateral and posterior approaches (Tables 1-3). The efficacy of both routes of injection was also supported by the statistically significant drop in the RMDI.

Comparison of efficacy with previous studies is difficult because there has been no standard defined for the appropriate number of injections or the period of follow-up. Slipman et $\mathrm{al}^{7}$ reported a good or excellent result in $60 \%$ of 20 patients based on an analysis of pain score, work status, medication use, and patient satisfaction. However, this result was based on patients receiving an average of 2.2 injections and follow-up ranging from 12 to 45 months. Multiple factors could thus have contributed to the overall improvement in these patients above and beyond the epidural therapy. Kolstad et $\mathrm{al}^{16}$ also performed multiple injections $(n=2)$ and demonstrated $>50 \%$ pain relief in $30 \%$ of patients at 6 weeks. Cyteval et al ${ }^{10}$ evaluated the pain response of 30 patients who underwent a single CT-guided cervical epidural injection with 6-month follow-up. Eighteen patients (60\%) demonstrated an excellent or good response ( $>50 \%$ pain reduction).

Various diagnostic tools have been used in an attempt to improve outcomes and localization of the level of cervical radiculopathy. These include the use of EMG, MR imaging, and preprocedural selective nerve blocks. ${ }^{8,16,17}$ Patients in this practice present for treatment after undergoing a variety of diagnostic tools, including CT, MR imaging, and EMG. Patients do not receive diagnostic nerve blocks before epidural injection. All patients referred for therapy have a current 

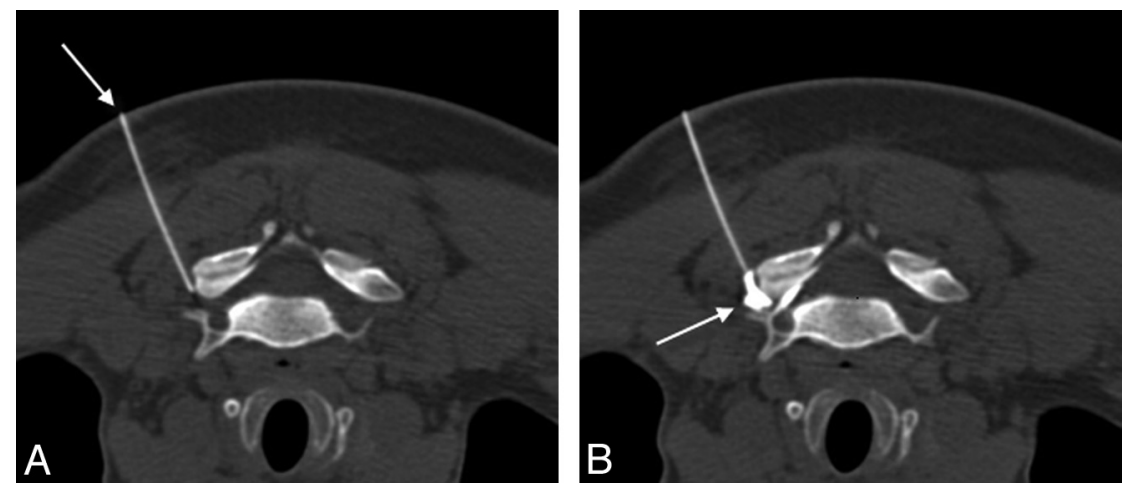

Fig 2. Posterior approach for CT-guided cervical epidural injection. A, Posterior placement of needle tip at the lateral margin of the C6-C7 neuroforamen. B, Periganglionic and central flow of contrast into the epidural space.

cross-sectional imaging study demonstrating varying degrees of foraminal or spinal canal encroachment at the proposed level of treatment. Clinical history and physical examinations are completed on all patients before epidural injection. The efficacy of CT-guided injections defined in this study, therefore, represents the response in a very diverse population without restriction by a specific preprocedural diagnostic work-up. These results are similar to previously published results defining the efficacy of cervical epidural injections.

Although there are numerous reports in the literature of adverse patient outcomes in cervical epidural injections, ${ }^{1,2,15,18}$ there were no significant complications in this retrospective series. Four vasovagal reactions were documented in 247 patients. These reactions were equally divided between the anterolateral and posterior groups. None of these reactions necessitated pharmacologic intervention. No other adverse outcomes were reported.

In considering the efficacy of the procedure, one can argue that the posterior approach does not allow optimal delivery of medication to the central epidural space because the needle approaches the exiting nerve at an acute angle. It is our observation that most of such injections will remain specific to the target nerve, with perineural flow outlining the dorsal root ganglion but with little penetration to the central epidural space. Hence, this approach is best used when diagnostic information is desired, and the pain pattern is thought to be referable to a specific single nerve. If the superior aspect of the foramen is targeted via a posterior approach, there is typically a fat plane adjacent to the facet joint, which will carry injected contrast and medication into the posterior foramen and ultimately into the epidural space (Fig $2 B$ ).

This study is limited by the retrospective nature of the review. To clearly define the efficacy of CT-guided epidural injections by using a posterior approach, a randomized prospective study would be needed. However, this would be a difficult study to perform given the typical levels of pain that patients are experiencing when they present for therapy. Few patients would want to be randomized to the control group. Kallmes et al recently defined the difficulty in recruiting patients when they are in pain and are presented with a therapy that has been defined by the medical community as being effective. ${ }^{19}$

The study is also limited by the percentage of patient follow-up data at the 2-week and 2-month phone interviews. Sixty percent of patients were contacted at 2 weeks, and $49 \%$ of patients were contacted at 2 months. The longitudinal mixed model was used to include all available data on any patient with at least 1 recorded VNPS or RMDI. The sensitivity analysis provided evidence that the 2-week and 2-month missing data mechanisms (missing completely at random and MAR) can be ignored. ${ }^{20}$ However, a higher percentage of data retrieval could certainly have resulted in minor changes in overall efficacy measures. An additional limitation of the study is that the small sample size precludes a comprehensive safety assessment. While no serious complications were observed, the upper limit of the $95 \%$ CI for the complication rate is as high as $1.2 \%$ according to the "rule of 3 " (3 divided by the sample size) ${ }^{21}$

Finally, this study does not define the long-term outcome of cervical epidural injections. It defines the safety and shortterm ( 8 weeks) efficacy of CT-guided transforaminal epidural injections by using a posterior approach in a patient population referred from multispecialty providers without restrictions on preprocedural evaluation. Different levels of efficacy could be defined if the patient population was restricted by specific preprocedural testing. Those studies may warrant future investigation.

\section{Conclusions}

CT-guided transforaminal cervical epidural injections by using a posterior approach are safe and effective. The efficacy of these injections is similar to that defined at 2 weeks and at 2 months by using the anterolateral approach in this study. The efficacy is also comparable with previous reports in the literature by using CT guidance or fluoroscopic guidance for transforaminal cervical epidural steroid administration.

\section{Acknowledgments}

We thank Angela Treadwell for her assistance with manuscript preparation.

\section{References}

1. Ludwig MA, Burns SP. Spinal cord infarction following cervical transforaminal epidural injection: a case report. Spine (Phila Pa 1976) 2005;30:E266-68

2. McMillan MR, Crumpton C. Cortical blindness and neurologic injury complicating cervical transforaminal injection for cervical radiculopathy. Anesthesiology 2003;99:509-11

3. Baker R, Dreyfuss P, Mercer S, et al. Cervical transforaminal injection of corticosteroids into a radicular artery: a possible mechanism for spinal cord injury. Pain 2003;103:211-15 
4. Wolter T, Knoeller S, Berlis A, et al. CT-guided cervical selective nerve root block with a dorsal approach. AJNR Am J Neuroradiol 2010;31:1831-36

5. Winnie AP, Hartman JT, Meyers HL Jr, et al. Pain clinic. II. Intradural and extradural corticosteroids for sciatica. Anesth Analg 1972;51:990-1003

6. Robecchi A, Capra R. Hydrocortisone (compound F): first clinical experiments in the field of rheumatology [in undetermined language]. Minerva Med 1952;43:1259-63

7. Slipman CW, Lipetz JS, Jackson HB, et al. Therapeutic selective nerve root block in the nonsurgical treatment of atraumatic cervical spondylotic radicular pain: a retrospective analysis with independent clinical review. Arch Phys Med Rehabil 2000;81:741-46

8. Slipman CW, Lipetz JS, DePalma MJ, et al. Therapeutic selective nerve root block in the nonsurgical treatment of traumatically induced cervical spondylotic radicular pain. Am J Phys Med Rehabil 2004;83:446-54

9. Castagnera L, Maurette P, Pointillart V, et al. Long-term results of cervical epidural steroid injection with and without morphine in chronic cervical radicular pain. Pain 1994;58:239-43

10. Cyteval C, Thomas E, Decoux E, et al. Cervical radiculopathy: open study on percutaneous periradicular foraminal steroid infiltration performed under CT control in 30 patients. AJNR Am J Neuroradiol 2004;25:441-45

11. Lasbleiz J, Siegfried D, Chales G, et al. Evaluation of CT guided cervical epidural injections in patients with mechanical cervicobrachial neuralgia [in French]. J Radiol 2008;89(3 pt 1):317-23
12. Lin EL, Lieu V, Halevi L, et al. Cervical epidural steroid injections for symptomatic disc herniations. J Spinal Disord Tech 2006;19:183-86

13. Stav A, Ovadia L, Sternberg A, et al. Cervical epidural steroid injection for cervicobrachialgia. Acta Anaesthesiol Scand 1993;37:562-66

14. Strobel K, Pfirrmann CW, Schmid M, et al. Cervical nerve root blocks: indications and role of MR imaging. Radiology 2004;233:87-92

15. Tiso RL, Cutler T, Catania JA, et al. Adverse central nervous system sequelae after selective transforaminal block: the role of corticosteroids. Spine J 2004;4:468-74

16. Kolstad F, Leivseth G, Nygaard OP. Transforaminal steroid injections in the treatment of cervical radiculopathy: a prospective outcome study. Acta Neurochir (Wien) 2005;147:1065-70, discussion 1070.

17. Anderberg L, Annertz M, Persson L, et al. Transforaminal steroid injections for the treatment of cervical radiculopathy: a prospective and randomised study. Eur Spine J 2007;16:321-28.

18. Akkaya T, Sayin M. Transforaminal epidural steroid injection and its complications [in Turkish]. Agri 2005;17:27-39

19. Kalllmes DF, Comstock BA, Heagerty PJ, et al. A randomized trial of vertebroplasty for osteoporotic spinal fractures. N Engl J Med 2009;361:569-79

20. Little RJA RD. Statistical Analysis with Missing Data. New York: John Wiley \& Sons; 2002.

21. Eypasch E, Lefering R, Kum CK, et al. Probability of adverse events that have not yet occurred: a statistical reminder. BMJ 1995;311:619-20 